\title{
"Buddy the saviour": Selected managerial strategies deployed to facilitate Infection Control in a hospital during the COVID-19 pandemic: Experience from the National Referral Centre of Sri Lanka
}

\author{
Attanayake H, Wijewickrama A, Idampitiya D, Athukorala S, Sooriarchchi C, \\ Udugamakorala G, Peramuna C, Karunartne M.
}

\begin{abstract}
Introduction: National Institute of Infectious Diseases (NIID) is the apex referral centre for clinical management of COVID-19 in Sri Lanka. It has remained resilient through twelve months from the onset of the pandemic. Infection Control was one of the prime concerns of its management.
\end{abstract}

Objective: The objective was to describe managerial strategies adopted by NIID to facilitate infection control during the COVID-19 pandemic.

Methods: This was a descriptive case study. Data was gathered through Key Informant Interviews and by examining hospital records.

Results: Training and improving awareness on infection control, ensuring good hand hygiene practices, management of Personal Protective Equipment (PPE), developing institutional capacities to ensure good infection control, effective cleaning and disinfection and prompt and proper management of staff outbreaks were the managerial strategies deployed by NIID to facilitate infection control.

Conclusions: Training and improving awareness on infection control, ensuring good hand hygiene practices, management of Personal Protective Equipment (PPE), developing institutional capacities to ensure good infection control, effective cleaning and disinfection and prompt and proper management of staff outbreaks are effective managerial strategies in facilitating infection control in a hospital setting.

Keywords: COVID-19, Infection Control, NIID

\section{Introduction}

National Institute of Infectious Diseases (NIID) is the National Focal Point for the clinical management of Infectious Diseases in Sri Lanka. It has managed patients with pandemic diseases such as Severe Acute Respiratory Syndrome (SARS), Avian Influenza and COVID-19 as of late.
Presently it serves as the Apex Referral Centre for clinical management of COVID-19 in Sri Lanka giving the clinical leadership to more than ninety (90) COVID-19 treatment centres island wide. As at 28.02.2021 NIID has treated 2703 COVID-19 positive patients, 1716 patients who were suspected of COVID-19; including 155 patients requiring critical care in the intensive care unit (ICU).

NIID has a bed strength of 150 for COVID-19 treatment (Resident Patient Care). It is comprised of two (2) units for single isolation, seven (7) units for cohort isolation and two ICUs.

NIID deploys 650 staff members as its workforce. This includes doctors, nurses, paramedics, health assistants, clerical staff and employees in the contractual services such as janitorial, laundry and security services.

As COVID-19 is a highly transmissible disease spread via droplet transmission mode (WHO, 2020) Infection Control was a challenge from the very beginning and was one of the top managerial concerns during the pandemic. The Infection Control of the hospital was monitored by the Infection Control Unit, The Infection Control Team (ICT) too played a vital role in trouble shooting and streamlining processes.

\section{Objective:}

To describe the managerial strategies adopted by NIID to facilitate infection control during the COVID-19 pandemic.

\section{Methods}

This was a descriptive case study. The period under the study was from 26.01.2020 up to 28.02.2021. Data was collected mainly through Key Informant Interviews (KII) and by examining Hospital Records. Data was entered both manually and electronically by the Principal Investigator (PI).

PI interviewed the four (4) Senior Consultant Physicians, two (2) Consultant Microbiologists, a Special Grade Nursing Officer (1), Infection Control Nursing Officer (1) and fifteen (15) in charge sisters/senior nurses in-charge of different units in KII. A semi structured interview schedule was used to collect data. The interview 
schedule covered core areas in infection control such as Hand hygiene, Personal Protective Equipment (PPE) $\&$ management of staff outbreaks. Subsequently three successive workshops were organized to synthesize, analyze and stabilize data gathered through KII. This improved the accuracy, validity and reliability of the gathered data.

\section{Results}

The following managerial strategies were deployed by NIID to facilitate infection control during the COVID-19 pandemic.

1. Training and improving awareness on infection control

2. Ensuring good hand hygiene practices

3. Management of Personal Protective Equipment (PPE)

4. Developing institutional capacities to ensure good infection control

5. Effective cleaning and disinfection

6. Prompt and proper management of staff outbreaks

Training and improving awareness on infection control Although the COVID-19 outbreak erupted in Sri Lanka in January 2020, the preparations for a possible pandemic disease were made following the announcement of Ebola as a public health emergency of international concern by the World Health Organization (WHO) in July 2019. A preparedness assessment was conducted and several recommendations were made in a view of improving institutional capacity. Training was one of the main concerns.

Doctors, nurses, paramedics and health assistants were scheduled for simulation drills conducted by the Planning Unit with the objective of improving knowledge and practices in core areas such as the use of personal protective equipment (PPE), standard precautions and occupational hazards. These simulations were videoed and reviewed by a panel of consultants. Gaps and mistakes were identified and communicated to the respective participants following the review. The final disaster management preparedness drill conducted in 05.09.2019 was evaluated by an external panel of experts. Following their comments certain protocols were revised. This enabled the staff to develop capacities and confidence even before the pandemic.

\section{Ensuring good hand hygiene practices}

Facilities for hand washing were improved for both staff and patients. Hand washing areas were established at the main entrance, entrance to clinic and OPD areas and in certain selected areas at the entrance to wards/units. Decanters with hand rub were placed by the side of the corridors with instructions to use in all three languages.

Hand hygiene is not only about facilities but also about practices. This aspect has been highlighted even before the dawn of the outbreak where a quality improvement project was launched in 2018 in a view of improving hand hygiene compliance (HHC) of the ICU.

\section{Figure 01 below shows the hand hygiene compliance among doctors, nurses and health assistants in the ICU in each quarter from 2018 through 2020.}

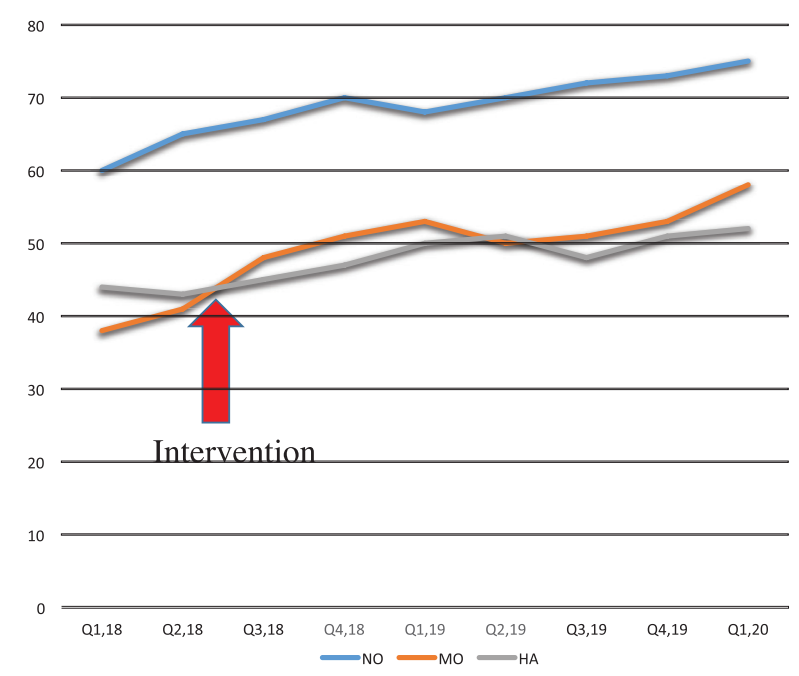

According to figure 01 , the $\mathrm{HHC}$ has been progressively improving in each staff category over the years following the intervention (quality improvement programme). This favoured the ICU to remain resilient having optimum infection control while treating large volumes of patients $(n=155)$ with COVID-19.

\section{Management of Personal Protective Equipment (PPE)}

Management of PPE is also of great importance in infection control. It has following aspects.

I. adequacy

There were at least nine (09) PPE items that were required to ensure protection against COVID-19. They were
a. overall/coverall/isolation gown
b. polythene apron
c. hair cap
d. mask/respirator
e. goggles or face shield
f. head cover
g. shoe covers
h. boots
i. gloves 
All PPE items (9) required per one health worker per one visit to the ward, were arranged as a "set". The requirement of these PPE sets varied according to the number of patients, criticality of patient illnesses, unit functions and deployed number of health workers to the respective unit. For the four (4) bedded ICU, 10-15 PPE sets were required per patient per day, which was the highest requirement. Should all resident patient care units be fully operational (including the two ICUs) the average requirement of PPE sets was one (1) per patient per day.

A dedicated team comprised of a Medical Officer, Matron and two Pharmacists were deployed to assess and monitor the use of PPE and forecast the fresh requirement according to the user pattern on a daily basis. This ensured the adequacy of PPE.

When there were disturbances to the supply chains, shortages were observed in many occasions. In such instances alternatives or donated items were used.

NIID maintained a stock of 200 PPE sets as a buffer to mitigate risk of shortages in surges.

II. Quality assurance and quality control

The specifications for PPE were prepared by a panel of consultants after studying both local and international guidelines. Comments and complaints made by end users were also considered in the formulation of specifications. As with donated items, several sample checks were done by a team comprised of the Consultant Microbiologist, Consultant Physician, Matron, Infection Control Nursing Officer and Pharmacist.

Filtration efficacy of the donated respirators were checked at Sri Lanka Institute of Nano Technology (SLINTEC).

\section{Storing}

Storing of PPE was also an important aspect in PPE Management. Once available stores were exhausted, insulated and air conditioned containers $(n=5)$ were used to store PPE. All PPE items were stored below $250 \mathrm{C}$.

IV. Continuous training and simulations

Training given to the staff at the preparatory phase was an investment as it prevented possible misuse/ overuse of expensive PPE items and thereby reduced wasting. But it was observed that certain staff members have got accustomed to their own techniques in donning and doffing PPE and have even developed "confidence" in doing so. As such clinical teams were invited for simulations in small groups. A volunteer from the group was requested to simulate donning/doffing and team along with an expert criticized the technique constructively. This enabled the staff members to realize the consequences by getting used to wrong/substandard techniques.

V. Close surveillance of donning and doffing of PPE Irrespective of the training any health worker may perform poor when it comes to donning and doffing due to anxiety, or mental/emotional stress. This was given thought from the very beginning. A" buddy" (a member from the same clinical team) was appointed to inspect the donning and doffing of PPE of a fellow team member and comment on his fine movements when performing duties.

Buddy was expected to be the helpful shadow of the health worker giving patient care, who escorted him up to the red zone (patient area). Buddy observed the movements of his counterpart being in the yellow zone (staff area) with adequate and appropriate PPE.

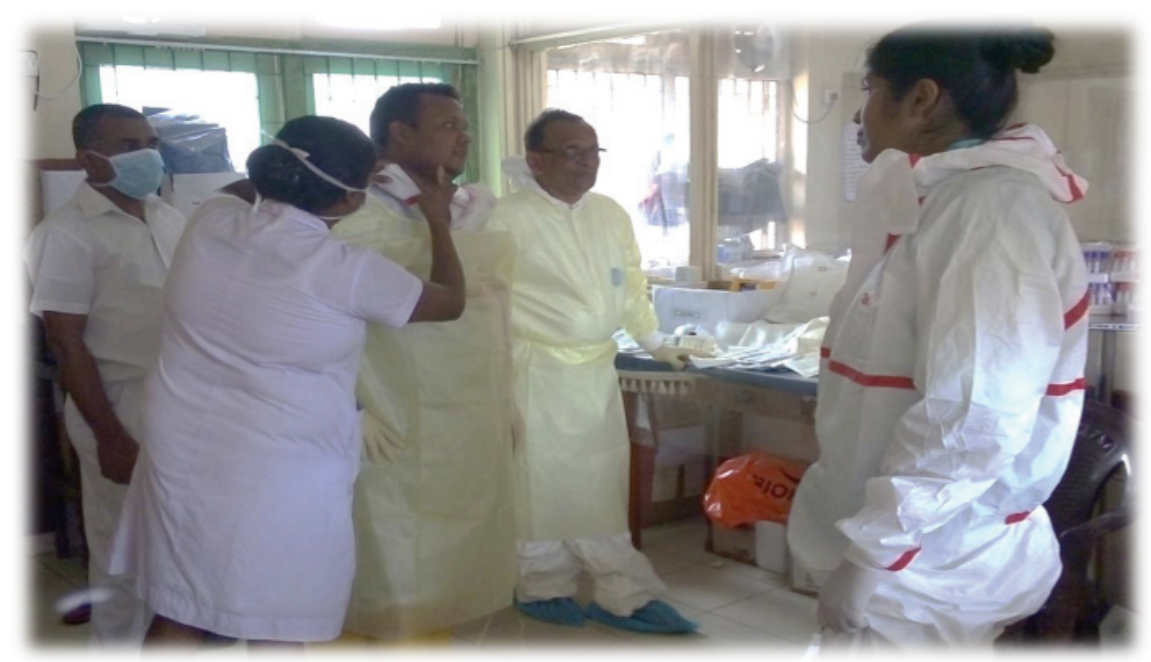

Figure 2: The Buddy inspecting the donning of a health worker. 
Figure 2 above is a photograph of the Buddy inspecting the donning of a health worker before he enters the isolation room.

\section{Developing institutional capacities to ensure good infection control}

A new unit of 16 rooms was put up strengthening the single isolation capacity. This enabled separation of positive patients from suspects.

The Mechanical Ventilation and Air Conditioning system (MVAC) of the Intensive Care Unit was renovated establishing the required negative pressure gradient. This enabled effective suction of the contaminated air in the isolation cubicle. High Efficiency Particulate Air (HEPA) filters were inserted to the air conducting system.

Additional air conditioners and air curtains were inserted to improve staff comfort as wearing nine items (9) of PPE for long hours itself was exhausting.

Transparent physical barriers were inserted in triage and reception areas separating patient from the care giver. Intercom facilities were provided where appropriate to communicate with the patient.

\section{Effective cleaning and disinfection}

Based on the recommendation by the Infection Control Committee, cleaning of the clinical units was removed from the out-sourced janitorial service and handed over to the Health Assistants under the supervision of the nursing staff. As this was done proactively before the pandemic which enabled effective cleaning during the pandemic.

Cleaning and disinfection was carried out as per national guidelines.

As few undesired effects of hypochlorite were observed when it was used as a disinfectant, a hydrogen peroxide vapourizer was used as an alternative especially in ICUs, isolation rooms and ambulances.

\section{Management of staff outbreaks}

Table 01 below shows the total number of staff members who became positive for COVID-19 in each quarter (an accumulative figure) during the period 26.01.2020-28.02.2021 and the corresponding number of co-workers who were quarantined (an accumulative figure) as a result of staff member becoming positive.

Table 01: Number of staff members positive and quarantined in 2020-21

\begin{tabular}{lrrr}
\hline & $\begin{array}{r}\text { Number of staff } \\
\text { members who } \\
\text { got positive for } \\
\text { COVID-19 (n=650) }\end{array}$ & $\begin{array}{r}\text { Number of staff } \\
\text { members who were } \\
\text { quarantined by } \\
\text { Risk assessment } \\
\text { committee }\end{array}$ & $\begin{array}{r}\text { Ratio of number of } \\
\text { positive staff members } \\
\text { : number of staff } \\
\text { members who were } \\
\text { quarantined }\end{array}$ \\
\hline 1st Quarter 2020 & 01 & 12 & $1: 12$ \\
2nd Quarter 2020 & 00 & 00 & - \\
3rd Quarter 2020 & 00 & 00 & - \\
4th Quarter 2020 & 07 & 43 & $1: 6$ \\
1st Quarter 2021 (up to 28.02.2021) & 17 & 40 & $1: 2$ \\
\hline Total & $\mathbf{2 5}$ & $\mathbf{9 5}$ & $\mathbf{1 : 4}$ \\
\hline
\end{tabular}

According to table 01, a total of 25 staff members has been positive for COVID-19 and 95 of them have been quarantined as a result in the said period. It is worth noting that the number of coworkers that required to be quarantined per one positive staff member was evidently becoming low even at such times the positive numbers were relatively high. This was probably due to the prompt and proper actions taken up by the management in such situations. Some of them are denoted below. 
I. Performing both Rapid Antigen Test (RAT) and PCR in all exposed health care workers as early as possible separating the non-infected from infected to curtail the spread

II. Decontaminating all staff areas of the unit

III. Re-emphasizing the importance of wearing masks, face shields, keeping distance, avoiding having meals/tea together and hand hygiene

IV. Providing adequate separate spaces for the staff to rest in rest hours

\section{Discussion}

Usually, the health worker is relatively safe at the point of the care delivery. But not in a pandemic. Nobody gets spared in a pandemic- neither the health worker nor the patient nor the visitor. As such Infection Control is one of the prime managerial concerns in a hospital during a pandemic. The top management has to come up with effective strategies innovatively to facilitate infection control. Strategies that are meant to educate, train, monitor, mentor and most of all supervise the infection control at the work front.

"The buddy" concept is a classic example of inserting intense supervision to a clinical unit in a more worker friendly approach. Buddy is not necessarily be a superior; nor a stranger, but a team member who observes the coworker in a view of preventing a possible exposure. Buddy is interchangeable and can even be the unit in charge when deploying a new comer.

The infection control team (ICT) cannot singly handle everything on its own during a pandemic. The management should liaise clinical units with the ICT to facilitate its functions.

\section{Conclusions}

Training and improving awareness on infection control, ensuring good hand hygiene practices, management of Personal Protective Equipment (PPE), developing institutional capacities to ensure good infection control, effective cleaning and disinfection and prompt and proper management of staff outbreaks are effective managerial strategies in facilitating infection control in a hospital setting during COVID-19 pandemic.

\section{References}

1. King, D., Vlaev, I., Everett-Thomas, R., Fitzpatrick, M., Darzi, A., \& Birnbach, D. J. (2016). "Priming" hand hygiene compliance in clinical environments. Health Psychology, 35(1), 96-101.
2. Von Lengerke, T., Lutze, B., Krauth, C., Lange, K., Stahmeyer, J. T., \& Chaberny, I. F. (2017). Promoting Hand Hygiene Compliance. Deutsches Arzteblatt international, 114(3), 29-36. doi:10.3238/arztebl.2017.0029

3. World Health Organization. Infection prevention and control of epidemic- and pandemic-prone acute respiratory infections in health care. Geneva: World Health Organization; 2014 Available from: https://apps.who.int/iris/bitstream/ handle/10665/112656/9789241507134_eng. pdf? sequence $=1$

4. World Health Organization \& WHO Patient Safety. (2009). WHO guidelines on hand hygiene in health care. World Health Organization. https://apps.who. int/iris/handle/10665/44102 